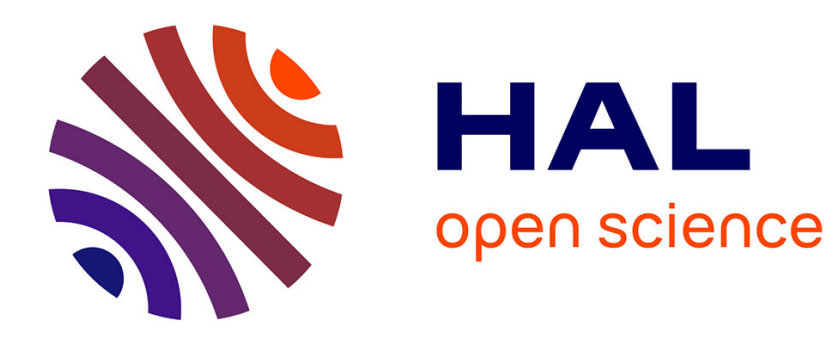

\title{
Disordering a superfluid of light
}

Matthieu Bellec, Claire Michel

\section{To cite this version:}

Matthieu Bellec, Claire Michel. Disordering a superfluid of light. Nature Photonics, 2020, 14, pp.471-

473. hal-03020453

\section{HAL Id: hal-03020453 \\ https://hal.science/hal-03020453}

Submitted on 23 Nov 2020

HAL is a multi-disciplinary open access archive for the deposit and dissemination of scientific research documents, whether they are published or not. The documents may come from teaching and research institutions in France or abroad, or from public or private research centers.
L'archive ouverte pluridisciplinaire HAL, est destinée au dépôt et à la diffusion de documents scientifiques de niveau recherche, publiés ou non, émanant des établissements d'enseignement et de recherche français ou étrangers, des laboratoires publics ou privés. 


\section{Subject: Nonlinear optics}

\section{Title: DISORDERING A SUPERFLUID OF LIGHT}

Standfirst: A phase transition between disordered and quasi-ordered states, known as Berezinskii-Kosterlitz-Thouless transition, has now been revealed in a two-dimensional photonic 'fluid'. The interplay between phase singularities and coherence may lead to new vortex-based optical applications.

Photonic devices utilizing integrated linear and nonlinear circuits, optical metamaterials and fibre-optics can be considered as low-dimensional systems. This arguably limits communication and information processing capabilities. Although efforts have been made to increase dimensions, through e.g. optical modal multiplexing, or 3D-nanoprinting techniques, there are technological limitations.

However, low-dimensionality is actually not always a restriction. 1D or 2D photonic devices can lead to rich and unexpected phenomena that may offer applications in both classical and quantum optics. For instance, topological photonics, a rapidly growing research field, is steeped in the 2D quantum Hall solid-state physics effect. Even the simpler topological photonic insulator 1D counterpart recently led to intense investigations. Feasible applications in robust optical transport, topological lasing and quantum communication have been proposed and realized [1].

Likewise, the Berezinskii-Kosterlitz-Thouless (BKT) transition [2] is another example showing that low dimensional condensed matter physics yields rich phenomenon. This nonconventional phase transition from disordered to quasi-ordered states in 2D systems, mediated by topological defects underlying dynamics, has been observed in quantum systems. Nonetheless, this transition mainly involves classical nonlinear wave dynamics.

Now writing in Nature Photonics, Situ and Fleischer reported the first observation of the BKT transition in optics, using a continuous laser in a crystal exhibiting a large optical nonlinear response, creating a 2D 'fluid of light' evolving along the propagation axis [3]. In their work, the associated topological defects are optical vortices, defined as phase singularities in the transverse optical wave field.

The science of structured light with singularities has already led to strong basic and applied scientific interests. Thus, besides the fundamental importance of this observation, the disclosed results, involving complex optical vortex dynamics, naturally appeal to potential applications in singularity-based photonics 2D devices [4].

An important property of low-dimensional physical systems (1D or 2D) is the absence of long range order, like e.g. crystalline, ferromagnetic or superfluid states, due to inherent thermal fluctuations at finite temperature. When short range interactions are additionally considered, Berezinskii and Kosterlitz/Thouless independently predicted in the 70s, that a phase transition between ordered and disordered states may occur in 2D systems [2]; it's usually said to be an unconventional transition, being continuous, mediated by topological defects, and without symmetry breaking. While 2D crystals or magnets are rather complicated to implement and 
study experimentally, two-dimensional fluids of interacting particles, possibly obeying BoseEinstein statistics, are much more accessible. Examples of such systems range from liquid Helium and superconductors to cold atoms and, more recently, quantum fluids of light. The order is then characterized by a high degree of phase coherence at long range, which is also called the superfluid state.

The role of the vortices in the BKT transition can be intuitively understood as follows. Consider the lowest energy state in a quantum fluid which has constant amplitude with flat phase. Adding a single vortex, defined by a wavefront spinning around a vanishing amplitude point, affects the field in a global way. At high temperatures, random motion of such vortices, which proliferate as the temperature increases, destroys large-scale phase coherence. If now two vortices spinning in opposite direction are paired together, which may occur as the temperature is decreased, the phase pattern is altered only locally and their circulation actually cancels, thus allowing long range order to appear. More precisely, the spatial phase correlations decrease exponentially in the disordered (normal) state and algebraically (power law) in the quasi-ordered (superfluid) state. At the BKT transition, which occurs at a critical temperature $T_{c}$, a universal jump in the superfluid density is predicted. The first experimental measurements of this jump took place soon after the BKT predictions in helium films [5]. In 2006, the transition was observed in ultracold atoms and first attempts to probe vortices dynamics were reported [6]. More recently, quantum fluids of light have been proposed as a rich alternative playground to quantitatively study advanced quantum hydrodynamics features [7], including superfluidity [7,8] and the BKT transition. Algebraic correlation functions were reported with exciton-polaritons in 2D semiconductor microcavities [9]. Inspired by a pioneering theoretical study [10], Situ and Fleischer characterize the optical BKT transition in a nonlinear optical setup allowing investigation of both the phase coherence features of the fluid of light, and the complex vortices underlying dynamics of the transition [3].

The experimental setup consists of a continuous laser beam propagating into an optical crystal with a large nonlinear photorefractive response (see Figure 1). As in other nonlinear media such as atomic vapors [11] or thermal liquids [12], under the paraxial approximation, the transverse evolution of the optical beam is analogous to a weakly interacting Bose-Einstein condensate (BEC). The propagation axis plays the role of time, while nonlinearities and refractive index modulation act as effective interactions and external potential. Such nonlinear optical dynamics is usually reformulated in terms of quantum hydrodynamics where the laser intensity and wavefront gradient correspond to the fluid density and velocity respectively. To deepen the analogy, a uniform wavefront scenario is typically analogous to a zero temperature BEC. Adding a controlled amount of random-phase components in the beam, achieved experimentally by using a spatial light modulator, allows increasing the condensate's effective temperature. This imposes density and phase fluctuations of the fluid of light, eventually leading to the generation of vortices, and enabling triggering of the BKT transition. In principle, noise alone should provoke the generation of the vortices, but in practice it is not sufficient. Here, a lattice is photo-induced in the photorefractive crystal, to help trigger vortices, as discussed below.

For each parameter set, varying the effective temperature and the strength and sign of the interaction, the output complex wavefunction (i.e. amplitude and phase of the optical field) is 
reconstructed via a standard interferometric technique. Two main quantities are then extracted to characterize the BKT transition: i) the number of new vortices that have been generated along the beam path along with their pairing status (vortices are considered as paired if their separation is smaller or equal to three vortex cores); ii) the radial correlation function. Its cumulative integral can actually be expressed, for all temperatures, $T$, simply as $\Omega^{2 \kappa(T)}$ where $\Omega$ is the integrating area and $\kappa$ is an exponent to be fitted. In theory, by looking at $\kappa$ as a function of $T$, the phase transition can be identified as a jump from $\kappa=0.875$ at just below a critical temperature $T_{c}$, to $\kappa=0.5$ just above $T_{c}$, corresponding to the algebraic (exponential) decay of the spatial correlations discussed above.

The presented experimental results show that the correlation functions obey the exact scaling behavior predicted by the BKT theory. The values of $\kappa$ are directly linked to the (universal) jump in the superfluid density. It's worth mentioning that the expected step function at $T_{c}$ was not observed; instead a smooth crossover was seen, an effect attributable to the finite size of the experimental system, which is an effect also observed in ultracold atoms experiments [6].

Above the critical temperature, the number of single free vortices that proliferate drastically increases. Counting them as function of $T$ allows to quantitatively obtain the value of $T_{c}$. Interestingly, the researchers observe that it actually depends on two parameters. Typical values of the normalized critical temperature range from 0.6 and 0.95 in accordance with reported results. First, $T_{c}$ is slightly shifted forward when the nonlinear strength is larger, whatever its sign. This is expected as the role of the density fluctuations is smaller in this case. Second, they measure a linear dependence of $T_{c}$ with the lattice spacing. A thermodynamics approach tells that smaller lattice spacings give more sites available for occupation (per a fixed area), increasing the entropy and indeed lowering the critical temperature needed to overcome the vortex energy. For a homogeneous system (i.e. when lattice spacing approaches zero) the transition should occur at finite $T_{c}$. What the researchers observe is, however, a wave condensation phenomenon with no evidence of vortex proliferation [13]. This raises the important issue of the role of the lattice in these experiments and the interplay between continuous and discrete dynamics. Situ and Fleischer found that the experimental parameter ranges are limited and the vortices can't be trigged by noise only (temperature fluctuations) in homogeneous fluids of light, thus requiring an additional lattice.

The results reported by Situ and Fleischer are of fundamental importance and constitute a starting point for exploring the full phase space of non-equilibrium and vortex-coherence dynamics in fluids of light. An interesting aspect to follow-up on may be the role of the lattice arrangement (with e.g. topological or disorder features) on the transition. Fluids of light thanks to the various and complementary existing photonic platforms - offer a rich playground to quantitatively study advanced quantum hydrodynamics features. Although restricted to 2D geometries, this work also shows that photonic systems generally exhibit complex dynamics with new light transport properties that may potentially lead to important applications in photonics.

Matthieu Bellec and Claire Michel

Institut de Physique de Nice, Université Côte d'Azur \& CNRS, Nice, France 


\section{References}

[1] Ozawa, T. et al. Rev. Mod. Phys. 9, 015006 (2019).

[2] Kosterlitz, J. M. \& Thouless, D. J. J. Phys. C 5, L124 (1972).

[3] Situ, G. \& Fleischer, J. W. Nat. Photon., xxx (2020).

[4] Soskin, M. et al. J. Opt. 19, 010401 (2016).

[5] Bishop, D. J. \& Reppy, J. D. Phys. Rev. Lett. 40, 1727 (1978).

[6] Hadzibabic, Z. et al. Nature 441, 1118-1121 (2006).

[7] Carusotto, I. and Ciuti, C. Rev. Mod. Phys. 85299 (2013).

[8] Michel, C. et al. Nat. Commun. 9, 2108 (2018).

[9] Roumpos, G. et al. Proc. Natl. Acad. Sci. 109, 6467-6472 (2012).

[10] Small, E. et al. Phys. Rev. A 83, 013806 (2011).

[11] Fontaine, Q. et al. Phys. Rev. Lett. 121, 183604 (2018).

[12] Vocke, D. et al. Optica 2, 484-490 (2015).

[13] Sun, C. et al. Nat. Phys. 8, 470-474 (2012).

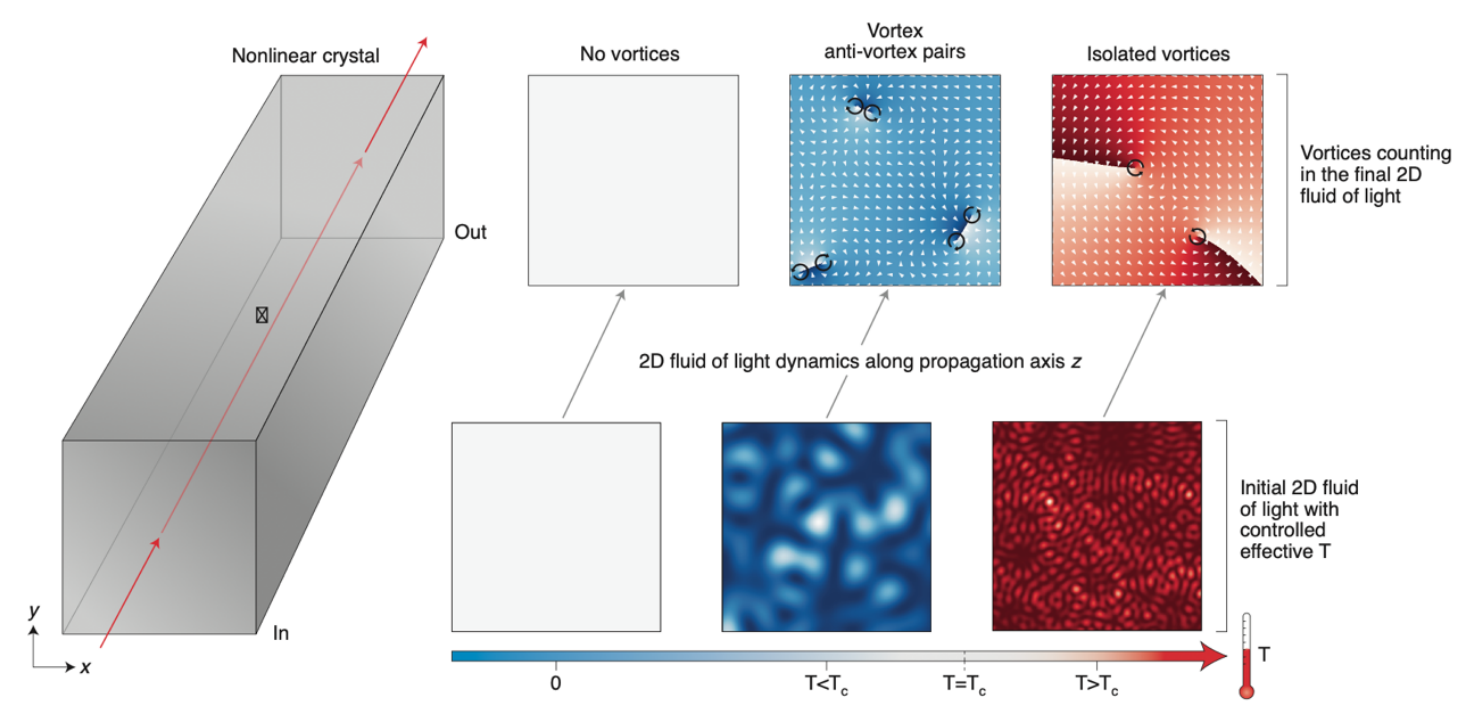

BKT transition in a fluid of light. Shining a laser on a nonlinear crystal allows to create a 2D 'fluid of light' evolving along the propagation $z$ axis (left). Random initial speckle controls the effective temperature of the system (bottom row). As the photonic fluid propagates, vortices are generated. A zoomed sketch of their phase distribution, usually measured at the output of the crystal, is presented (top row). At low temperature vortices are always paired. The phase coherence is affected locally only. This is a quasi-ordered state. Above the critical temperature, free vortices proliferate. The phase coherence is affected globally thus destroying the order. 